\title{
Amino acid and protein modification by oxygen and nitrogen species
}

\author{
Francesco Galli
}

Published online: 3 July 2010

(C) Springer-Verlag 2010

\section{Editorial preface}

This is the third special issue dedicated to "Amino acid and protein modification by oxygen and nitrogen species" that is now available for the readers of this journal and all the scientific community.

The first two very successful editions were published in vol 25, nos. 3-4, 2003 and vol 32, no. 4, 2007, with the contribution of such prestigious Guest Editors as Earl Stadtman, Rafael Radi and Victor Darley-Usmar. To continue with this tradition of excellence, on this occasion, an outstanding Board of Guest Editors has also been invited to promote this editorial initiative and recruit a selected panel of authors, many of who participated as speakers in the session dedicated to this subject at the 11th ICAAP conference held in Vienna, Austria, 3-7 August 2009. In fact, several other sessions in this conference included presentations on the subject of reactive oxygen species and their interactions with proteins and amino acids (see the abstract book published in vol 37, Suppl 1, 2009 of this journal). Once again this illustrates the widespread interest of researchers in this aspect of protein science, as well as the impressive literature that is dedicated to this subject every year. The fact that this remains a topic of mainstream interest was also highlighted by the Professor Emeritus Helmut Sies (founder of the "oxidative stress" concept) who revisited fundamental scientific and historical moments in the field during his plenary lecture on "oxidative stress" held at ICAAP 2009.

\section{F. Galli $(\square)$}

Laboratory of Biochemistry and Nutrition,

Department of Internal Medicine, School of Pharmacy,

University of Perugia, Via del Giochetto, 06126 Perugia, Italy

e-mail: f.galli@unipg.it
The thematic papers ${ }^{1}$ contributed by the invited authors in this volume of Amino Acids touch different aspects of reactive oxygen and nitrogen species (ROS and RNS, respectively) and their relationship with protein chemistry and biology.

As Field Editor, I am proud to promote and be part of this initiative, which was supported in prims by the Editorin-Chief and all the Board of Editors and Reviewers, all of whom did valuable and serious work during the peerreviewing process. I would like to thank all of them, but particularly the Guest Editors Tilman Grune, Ines BatinicHaberle and Daniela Salvemini for their patience and assiduous contribution during this collaboration.

\section{Editorial}

During the last few years, a conspicuous number of studies have focused on analysis platforms and functional aspects of proteomic investigation of post-translational modifications (oxPTMs). This has revealed innovative concepts and protocols that are now also available for the systematic study of reactive oxygen and nitrogen species (ROS and RNS, respectively)-derived PTMs (oxPTMs) within the new era of redox-proteomics (Dalle-Donne et al. 2005; Sultana et al. 2006). This aspect is discussed in a first group of review papers published in this special issue.

The oxPTMs form on amino acid residues during the life of a protein as a consequence of physiological regulatory or damage events, and is now well established that ROS and RNS are capable of generating them via targeted and pathway-specific reactions. As one of the most relevant

\footnotetext{
${ }^{1}$ All the review manuscripts in this issue have been subjected to external peer-reviewing according to the policy of this journal.
} 
consequences resulting from this aspect of protein chemistry, Drs. Spickett and Pitt (2010) described in their comprehensive review paper the role of oxPTMs in cell signaling and transcriptional regulation of genes. As well as oxidations of cysteine and methionine, modifications of amino acids such as proline, asparagine and tyrosine are gaining ground in the signaling field, and improved analytical tools and interpretative models are steadily enlarging the list of signaling proteins responding to redoxdependent regulatory events. In this context, modern mass spectrometers and laboratory protocols provide specific strategies to identify ROS/RNS derived-PTMs and their localization within the protein structure, and this remains the ultimate and most important level of evidence in molecular diagnostics of oxPTMs. While labeling approaches to detecting sulfur oxidations are well established, $\mathrm{MS}^{2}$ and $\mathrm{MS}^{3}$ methods for detecting other oxPTMs are now being developed.

Proteomics data handling and functional interpretation are another critical aspect of redox-proteomics that need further advancement. In this special issue, the review paper by Dr. Allmer (2010) describes bioinformatic platforms and subsidiary tools for qualitative and quantitative evaluation of proteomics data with PTMs produced by means of current proteomics technology, particularly during high throughput mass spectrometric analyses. A special focus of the review is on non-commercial data analysis tools that can either be used or adapted to quantitate PTMs among samples from LC-MS or LC-MS ${ }^{x}$ measurements.

Once modified, protein residues change their biochemical and physical behavior within the protein structure which may produce the structural and functional consequences that have been documented on a range of protein targets and biochemical processes such as enzymatic catalysis, cytoskeletal assembly and solute transport, cell metabolism and signaling, and protein turnover (Stadtman and Levine 2003). Only in a few cases, oxidation reactions form PTMs that are reversible, reparable or compatible with normal structure-function of a given protein; more often oxidative modifications produce stable covalent structures that, in addition of the oxPTMs themselves, result in intra- and inter-molecular cross-links, and formation of peptides and protein byproducts, as well as immunogenic (non-self) epitopes. All these levels of damage can represent discrete signals for protein removal. In this issue, the review paper by Grimm et al. (2010) describes these aspects in relation to protein metabolism and turnover by the proteasome. Although studied for quite a while, the mechanism of removal of oxidized proteins is still a matter of debate. This concerns basic questions such as the proteolytic systems involved, the mechanisms of recognition for the oxidized substrates, interactions with the chaperone system in the cell and many others.
Other types of oxPTMs include for instance a series of protein carbonyls derived from lipoxidation and glycation reactions that affect mostly Lys and Arg residues and also series of Pro and His oxidation products; these have been described in detail in two previous issues of this series (vol 25, nos. 3-4, 2003 and vol 32, no. 4, 2007). The products derived from the modification of Cys in proteins remain of particular interest, in view of the exquisite biological relevance that this residue has in protein structure-function and metabolism (Giles et al. 2003; van Montfort et al. 2003; Nakajima et al. 2007; Zumbrennen et al. 2009).

A very interesting contribution was made by Nauser et al. (2010) which aimed to further our knowledge about why nature uses selenium in the active site of the major cellular reductants, glutathione peroxidases (GPx) and thioredoxin reductases (TrxR). When selenocysteine is replaced with cysteine, the enzyme GPx is less active. The authors gave evidence, via kinetic and thermodynamic considerations, that there may be several reasons for the revolutionary selection of selenium. The one-electron oxidized product, selenyl radical, is considerably less oxidizing when compared to the cysteine radical: hydrogen abstraction from a protein backbone can practically be excluded with the selenyl radical. Furthermore, the reason may also lie in the tuning of the redox potential and/or hydrophilicity related to the differences in selenocysteine deprotonation as at physiological $\mathrm{pH}$ selenocysteine is fully deprotonated.

The oxPTMs deriving from the reaction with aromatic residues, and particularly with tyrosine, are of particular interest. These include NO-derived reactions with proteins, which appear to have important functional consequences (Bartesaghi et al. 2007; Souza et al. 2008; Ischiropoulos 2009). In this special issue, two manuscripts have described biological and methodological aspects concerning the analysis of protein-bound 3 '-nitrotyrosine. The review paper of Tsikas (2010) provides an accurate examination of the literature on mass spectrometry analysis of $3^{\prime}$-nitrotyrosine. Although this PTM has been extensively investigated in body fluids and solid tissues as an inflammatory and oxidative stress biomarker in age-related conditions such as neurodegeneration, atherothrombotic cardiovascular disease, diabetes and uremia (Sultana et al. 2006; Galli 2007; Peluffo and Radi 2007), the accurate (unbiased) analysis of $3^{\prime}$-nitrotyrosine in proteins is still an analytical challenge (Spickett et al. 2006; Abello et al. 2009; Chiappetta et al. 2009). Tandem mass spectrometry represents a reference technology for the study of $3^{\prime}$-nitrotyrosine in biological systems.

The review paper by Dr. Aslan (2010) has examined functional aspects concerning actin tyrosine nitration focusing on both in vitro and in vivo aspects of redoxproteomics of this specific nitroprotein that could be 
relevant to biomarker analysis and disease mechanism interpretation.

A second series of papers published in this special issue include contributions that describe the most relevant literature on endogenous and exogenous antioxidants. While for mechanistic purposes endogenous antioxidants could be overexpressed, such strategies would be impractical for therapeutic purposes; thus, synthetic antioxidants have been actively sought for over two decades. Holley et al. (2010) provide a comprehensive review on the key role of the endogenous antioxidant MnSOD under physiological and pathological conditions, in particular in cancer. Life is not possible without MnSOD. Its levels are greatly decreased in early stage cancer. Its overexpression reduces tumor growth, but its reduction does not facilitate tumor formation. Its modes of actions are complicated by the fact that it acts both via anti-proliferative and anti-apoptotic pathways, which appear to be associated with distinct biochemical mechanisms. In fact, anti- and pro-oxidative mechanisms have been suggested for these actions. The former involve decreasing levels of superoxide and its products, thus suppressing cellular transcriptional activity, and the latter works by the cytotoxic action of $\mathrm{H}_{2} \mathrm{O}_{2}$ produced via superoxide dismutation.

The critical role of reactive species and MnSOD was further justified in the article on pain by Little et al. (2010). The authors summarize the current and rapidly expanding knowledge of the roles of superoxide and peroxynitrite in acute and chronic neuropathic and non-neuropathic pain as well as in the development of pain associated with the prolonged use of opiates such as morphine. Thus the potential use of SOD mimics and peroxynitrite scavengers as novel non-narcotic analgesics is justified. In their models they clearly show that in chronic pain, besides the modification of critical proteins involved in neurotransmission (glutamate transferase and glutamine synthase), MnSOD (but not $\mathrm{Cu}, \mathrm{ZnSOD}$ ) was also oxidatively modified; specifically the nitration of protein tyrosine was observed.

Batinic-Haberle et al. (2010) summarized the design of Mn porphyrin-based antioxidants aimed at decreasing oxidative stress injuries. Nature has used porphyrins as active sites of critical endogenous proteins and enzymes such as hemoglobin, myoglobin, nitric oxide synthases, cyt P450, cyclooxygenases, oxidases, guanylyl cyclase, etc.; the porphyrin ligand preserves the integrity of the metal site where respective reactions occur. Thus, modifying porphyrin is a natural strategy to provide a metal site that can effectively scavenge superoxide, peroxynitrite, carbonate radical, hypochlorous acid, etc., and therefore (1) decrease oxidative damage of proteins, lipids and nucleic acids and (2) modulate cellular transcriptional activity. The latter may include the oxidative modification of signaling proteins, in particular NF- $\kappa \mathrm{B}$. In turn, $\mathrm{Mn}(\mathrm{III})$ cationic
$N$-substituted pyridylporphyrins are able to suppress excessive inflammatory and immune responses in a variety of diseases. Importantly, cationic porphyrins substituting there for MnSOD, accumulate significantly in mitochondria. Effects observed with Mn porphyrins are similar to the effects observed with MnSOD-overexpressed mice. The wealth of in vivo data that justifies the design strategies is detailed.

If the $\mathrm{Mn}$ site in a cationic $N$-alkylpyridylporphyrin is replaced with $\mathrm{Zn}$, a potent photosensitizer emerges. Mn and $\mathrm{Zn}$ porphyrins influence oxidative stress in opposing ways. While Mn porphyrins alone predominantly scavenge ROS, $\mathrm{Zn}$ porphyrins afford therapy via production of reactive species. Benov et al. (2010) summarized the photodynamic effects of $\mathrm{Zn}$ porphyrins in cellular systems (both prokaryotic Escherichia coli and eukaryotic mammalian cell types, in particular cancer cell lines) in comparison to clinically employed hematoporphyrin D (Photofrin). The authors show that $\mathrm{Zn}$ porphyrins can inhibit major antioxidant, metabolic and mitochondrial enzymes and membrane proteins. The authors also showed how modification of the porphyrin structure enhances their lipophilicity, in turn bioavailability and thus their in vivo effects. Again, the cationic $\mathrm{Zn}$ porphyrins, and most so lipophilic ones like their Mn analogs, distribute in mitochondria which enhances their cell-killing actions.

The Guest Editors express their appreciation for the outstanding contribution of the invited authors and for the support of the Board of Editors of this journal and also of the reviewers who provided valuable work during all the phases of production of this special issue.

\section{Guest Editors}

Tilman Grune

Institute of Nutrition, Friedrich Schiller University, Jena, Germany

Ines Batinic-Haberle

Department of Radiation Oncology, Duke University Medical Center, Durham, NC 27710, USA

Daniela Salvemini

Department of Pharmacological and Physiological Science, Saint Louis University School of Medicine, St Louis, MO, USA

Francesco Galli

Department of Internal Medicine, School of Pharmacy, University of Perugia, Perugia, Italy

\section{References}

Abello N, Kerstjens HA et al (2009) Protein tyrosine nitration: selectivity, physicochemical and biological consequences, denitration, and proteomics methods for the identification of tyrosine-nitrated proteins. J Proteome Res 8(7):3222-3238 
Allmer J (2010) Existing bioinformatics tools for the quantitation of post-translational modifications. Amino Acids (in this issue). doi:10.1007/s00726-010-0614-3

Aslan M (2010) Functional consequences of actin nitration: in vitro and in disease states. Amino Acids (in this issue). doi:10.1007/ s00726-010-0613-4

Bartesaghi S, Ferrer-Sueta G et al (2007) Protein tyrosine nitration in hydrophilic and hydrophobic environments. Amino Acids 32(4):501-515

Batinic-Haberle I, Spasojevic I, Tse HM, Tovmasyan A, Rajic Z, St Clair DK, Vujaskovic Z, Dewhirst MW, Piganelli JD (2010) Design of Mn porphyrins for treating oxidative stress injuries and their redox-based regulation of cellular transcriptional activities. Amino Acids (in this issue). doi:10.1007/s00726-0100603-6

Benov L, Craik J, Batinic-Haberle I (2010) Protein damage by photoactivated $\mathrm{Zn}$ (II) $\mathrm{N}$-alkylpyridylporphyrins. Amino Acids (in this issue). doi:10.1007/s00726-010-0640-1

Chiappetta G, Corbo C et al (2009) Quantitative identification of protein nitration sites. Proteomics 9(6):1524-1537

Dalle-Donne I, Scaloni A et al (2005) Proteins as biomarkers of oxidative/nitrosative stress in diseases: the contribution of redox proteomics. Mass Spectrom Rev 24(1):55-99

Galli F (2007) Protein damage and inflammation in uraemia and dialysis patients. Nephrol Dial Transplant 22(Suppl 5):v20-v36

Giles NM, Watts AB et al (2003) Metal and redox modulation of cysteine protein function. Chem Biol 10(8):677-693

Grimm S, Höhn A, Grune T (2010) Oxidative protein damage and the proteasome. Amino Acids (in this issue). doi:10.1007/s00726010-0646-8

Holley AK, Dhar SK, Xu Y, St Clair DK (2010) Manganese superoxide dismutase: beyond life and death. Amino Acids (in this issue). doi:10.1007/s00726-010-0600-9

Ischiropoulos H (2009) Protein tyrosine nitration-an update. Arch Biochem Biophys 484(2):117-121

Little JW, Doyle T, Salvemini D (2010) Reactive nitroxidative species and nociceptive processing: determining the roles for nitric oxide, superoxide, and peroxynitrite in pain. Amino Acids (in this issue). doi:10.1007/s00726-010-0633-0

Nakajima H, Amano W et al (2007) The active site cysteine of the proapoptotic protein glyceraldehyde-3-phosphate dehydrogenase is essential in oxidative stress-induced aggregation and cell death. J Biol Chem 282(36):26562-26574

Nauser T, Steinmann D, Koppenol WH (2010) Why do proteins use selenocysteine instead of cysteine? Amino Acids (in this issue). doi:10.1007/s00726-010-0602-7

Peluffo G, Radi R (2007) Biochemistry of protein tyrosine nitration in cardiovascular pathology. Cardiovasc Res 75(2):291-302

Souza JM, Peluffo G et al (2008) Protein tyrosine nitrationfunctional alteration or just a biomarker? Free Radic Biol Med 45(4):357-366

Spickett CM, Pitt AR (2010) Protein oxidation: role in signalling and detection by mass spectrometry. Amino Acids (in this issue). doi: 10.1007/s00726-010-0585-4

Spickett CM, Pitt AR et al (2006) Proteomic analysis of phosphorylation, oxidation and nitrosylation in signal transduction. Biochim Biophys Acta 1764(12):1823-1841

Stadtman ER, Levine RL (2003) Free radical-mediated oxidation of free amino acids and amino acid residues in proteins. Amino Acids 25(3-4):207-218

Sultana R, Boyd-Kimball D et al (2006) Redox proteomics identification of oxidized proteins in Alzheimer's disease hippocampus and cerebellum: an approach to understand pathological and biochemical alterations in AD. Neurobiol Aging 27(11):1564-1576

Tsikas D (2010) Analytical methods for 3-nitrotyrosine quantification in biological samples: the unique role of tandem mass spectrometry. Amino Acids (in this issue). doi:10.1007/s00726010-0604-5

van Montfort RL, Congreve M et al (2003) Oxidation state of the active-site cysteine in protein tyrosine phosphatase 1B. Nature 423(6941):773-777

Zumbrennen KB, Wallander ML et al (2009) Cysteine oxidation regulates the RNA-binding activity of iron regulatory protein 2 . Mol Cell Biol 29(8):2219-2229 